\title{
BMJ Open Antiepileptic drugs and the risk of ischaemic stroke and myocardial infarction: a population-based cohort study
}

\author{
Christel Renoux, ${ }^{1,2,3}$ Sophie Dell'Aniello, ${ }^{1}$ Olli Saarela, ${ }^{4}$ Kristian B Filion, ${ }^{1,3,5}$ \\ Jean-François Boivin ${ }^{1,3}$
}

To cite: Renoux C,

Dell'Aniello S, Saarela 0, et al. Antiepileptic drugs and the risk of ischaemic stroke and myocardial infarction: a population-based cohort study. BMJ Open 2015;5: 0008365

doi:10.1136/bmjopen-2015008365

- Prepublication history and additional material is available online. To view please visit the journal (http://dx.doi.org/ 10.1136/bmjopen-2015008365)

Received 30 March 2015 Revised 18 June 2015 Accepted 17 July 2015



For numbered affiliations see end of article.

Correspondence to Professor Christel Renoux; christel.renoux@mcgill.ca

\section{ABSTRACT}

Objectives: Hepatic enzyme-inducing antiepileptic drugs (AEDs) increase serum lipid levels and other atherogenic markers via the induction of cytochrome P450 and may therefore increase the risk of vascular events. We sought to assess the risk of ischaemic stroke and myocardial infarction (MI) according to AED enzymatic properties.

Design: Population-based cohort study with nested case-control analysis.

Setting: 650 general practices in the UK contributing to the Clinical Practice Research Datalink.

Participants: A cohort of 252407 incident AED users aged 18 or older between January 1990 and April 2013. For each case of ischaemic stroke or MI, up to 10 controls were randomly selected among the cohort members in the risk sets defined by the case and matched on age, sex, indication for AED, calendar time and duration of follow-up.

Interventions: Current use of enzyme-inducing and enzyme-inhibiting AEDs compared with non-inducing AEDs.

Primary outcome measures: Incidence rate ratios (RRs) of ischaemic stroke and MI.

Results: 5069 strokes and 3636 Mls were identified during follow-up. Inducing AEDs use was associated with a small increased risk of ischaemic stroke ( $R R=1.16$, $95 \% \mathrm{Cl} 1.02$ to 1.33 ) relative to non-inducing AEDs, most likely due to residual confounding. However, current use of inducing AEDs for $\geq 24$ months was associated with a $46 \%$ increased risk of $M I(R R=1.46$, $95 \% \mathrm{Cl} 1.15$ to 1.85 ) compared with the same duration of non-inducing AED, corresponding to a risk difference of $1.39 / 1000$ (95\% Cl 0.33 to 2.45$)$ persons per year. Current use of inhibiting AED was associated with a decreased risk of $\mathrm{MI}(\mathrm{RR}=0.81,95 \% \mathrm{Cl} 0.66$ to 1.00$)$.

Conclusions: The use of enzyme-inducing AEDs was not associated with an increased risk of ischaemic stroke; a small increase of MI with prolonged use was observed. In contrast, use of inhibiting AEDs was associated with a decreased risk of MI.

\section{INTRODUCTION}

Antiepileptic drugs (AEDs) are increasingly used to treat conditions other than epilepsy

\section{Strengths and limitations of this study}

- We conducted a large population-based cohort study using the UK Clinical Practice Research Datalink (CPRD), allowing precise estimates and generalisability of our results.

- We estimated rates of vascular events within groups of patients with the same indication for antiepileptic drugs, minimising the potential for confounding by indication.

- Results were consistent when using alternative methods to control for confounding.

- Exposure to antiepileptic drugs was based on prescriptions issued by physicians and not on prescriptions actually filled or taken by patients.

such as migraine, pain originating from the nervous system or bipolar disorders. As such, it is estimated that more than $1 \%$ of the general population is exposed to AEDs. ${ }^{1}$ AEDs can be distinguished according to their action on the liver enzymatic system as inducing AEDs, non-inducing AEDs and inhibiting AEDs. ${ }^{2}$ Older AEDs, that is, carbamazepine, phenobarbital, phenytoin and primidone are all inducing AEDs, except sodium valproate, which is the only inhibiting AED. Second-generation AEDs have weak or non-inducing properties. ${ }^{2}$

Through their potent hepatic enzyme-inducing properties, predominantly on the cytochrome P450 system, inducing AEDs may lead to drug interactions and alter various metabolisms, including lipid metabolism. ${ }^{3}$ Indeed, several studies have shown that adults with epilepsy treated with inducing AEDs have increased serum levels of total cholesterol, low-density lipoprotein (LDL) cholesterol, triglycerides, lipoprotein (a), as well as $\mathrm{C}$ reactive protein and homocysteine. ${ }^{4-7}$ Similar findings were observed in healthy users exposed to carbamazepine ${ }^{4}$ and these changes are detectable 2-3 months after 
onset of treatment. ${ }^{4-8}$ Moreover, switching from inducing AEDs to non-inducing AEDs led to a significant decrease in these markers of atherothrombotic risk. ${ }^{6-9}$ Since enzyme-inducing AEDs promote proatherogenic factors, concerns have been raised regarding their use as first-line agents in the absence of studies properly assessing their effect on the risk of cardiovascular and cerebrovascular events. ${ }^{10}$ Conversely, sodium valproate, which possesses inhibiting enzymatic properties, has not been associated with such metabolic changes. However, the net vascular effect of sodium valproate is unclear as valproate could have a proatherogenic effect through induction of insulin resistance, body weight gain, metabolic syndrome and increased oxidative stress. ${ }^{11} 12$

Several cross-sectional studies have shown that patients with epilepsy have a higher prevalence of vascular risk factors and vascular diseases than the general population. ${ }^{13}$ Considering the increasing use of AEDs, and the long-term exposure of patients in the context of chronic diseases, a thorough investigation of their vascular risk is warranted. The objective of this study was therefore to assess separately the risk of ischaemic stroke and myocardial infarction (MI) associated with the use of inducing and inhibiting AEDs compared with non-inducing AEDs using a population-based cohort of patients newly treated with AEDs.

\section{METHODS}

\section{Data source}

The source population was identified using the UK's Clinical Practice Research Datalink (CPRD; previously known as General Practice Research Database (GPRD) ), ${ }^{14} 15$ the world's largest computerised database of anonymised primary care medical records on a population of over 12 million patients enrolled with more than 650 general practices. The geographic distribution of the practices, as well as age and sex distributions of patients, broadly reflects those of the UK population. ${ }^{14}$ Information collected includes demographic characteristics, lifestyle factors, medical symptoms and diagnoses, laboratory tests, prescriptions and referrals to specialists and hospitals. Prescriptions dispensed by the general practitioner are automatically recorded on the computerised file. Read codes are used to enter medical diagnoses ${ }^{16}$ and a coded drug dictionary based on the UK Prescription Pricing Authority Dictionary is used for recording prescriptions. General practitioners are specifically trained for the recording of data, and practices fulfilling predefined quality criteria are labelled 'up-to-standard' (UTS). ${ }^{17}$ Completeness and quality of data as well as consistency with medical files are checked regularly, and numerous studies have shown the high quality of recorded data, ${ }^{18} 19$ including diagnostic codes for stroke and MI. ${ }^{20}$

\section{Study design and cohort definition}

We conducted a retrospective cohort study that was analysed using a nested case-control approach, ${ }^{21}{ }^{22}$ separately for the two outcomes. This nested casecontrol approach is computationally more efficient than a time-dependent survival analysis while producing equivalent estimates. ${ }^{23} 24$ The cohort was formed of all patients in the CPRD aged 18 years or older who were members of a UTS practice, and who received at least one prescription for one of the following AEDs between 1 January 1990 and 30 April 2013: beclamide, carbamazepine, ethosuximide, gabapentin, lacosamide, lamotrigine, levetiracetam, oxcarbazepine, phenobarbital, phenytoin, piracetam, pregabalin, primidone, rufinamide, sodium valproate, tiagabine, topiramate, vigabatrin and zonisamide. Cohort entry (time zero) was taken as the date of the first AED prescription within the study period. Patients with less than 1 year of information in the database prior to the date of cohort entry were excluded, as were patients who had been dispensed an AED before cohort entry in order to include only new users of AEDs. ${ }^{25}$ Patients with a history of stroke at any time before cohort entry were excluded when studying stroke, whereas patients with a history of MI were excluded when studying MI. Patients were followed until the date of their first stroke or MI, depending on the outcome under study, the date they transferred out of the UTS practice, death or end of the study period (30 April 2013), whichever occurred first.

\section{Case definition}

For both outcomes, we identified all participants within our cohort with a first-time diagnostic code recorded for stroke or MI after cohort entry. The index date was defined as the calendar date of the first recorded ischaemic stroke, or MI, depending on the outcome under study. Potential cases of stroke were identified using codes referring to ischaemic, haemorrhagic and unspecified types of strokes (eg, cerebrovascular accident). An algorithm integrating examinations, procedures and treatments was developed to classify strokes not further specified as ischaemic or haemorrhagic. The computerised medical records of a random sample $(10 \%)$ of the potential cases of stroke were reviewed by a neurologist (CR), blinded to exposure status, and the corresponding algorithm was modified until complete agreement between the computer and the reviewer's diagnostic classification was achieved. Following this process, strokes whose type could not be determined $(30.6 \%)$ were classified as ischaemic strokes. Patients with a first code for haemorrhagic stroke were censored at the date of diagnosis and only ischaemic strokes and strokes not otherwise specified were considered as cases.

\section{Control selection}

For each case, up to 10 controls were randomly selected among the cohort members in the risk sets defined by the case, after matching on sex, age $( \pm 1$ year $)$, indication for AED prescription (epilepsy, pain, psychiatric disease, other/unknown), date of cohort entry $( \pm 1$ year $)$ and 
duration of follow-up. When no controls were found for a case, matching criteria were relaxed for cohort entry ( \pm 2 years) and for age ( \pm 5 years). The matching on calendar time permits controlling for trends over time in the use of AEDs and changes in incidence of the outcome, whereas matching on indication of AED is essential to control for the baseline vascular risk that is likely to differ between indications. Controls being selected from the risk set defined by each case, they were necessarily alive, active in the practice and eventfree when matched to their corresponding case. The date resulting in the same duration of follow-up for the case and controls defined the index date for the controls.

\section{Definition of AED exposure}

For each matched set of cases and controls, all AED prescriptions issued between cohort entry and the index date were identified. The duration of each prescription was calculated from the number of prescribed tablets combined with dosage instructions. In order to assess if the risk of ischaemic vascular event varies according to enzyme-inducing properties, AEDs were classified into the three following classes: inducing AED (carbamazepine, phenytoin, phenobarbital and primidone), inhibiting AED (sodium valproate) and non-inducing AED (all other AEDs). Patients were considered current users if the duration of their last prescription included the index date or ended within 90 days prior to the index date. We further distinguished between those who had no AED prescription in the year before the index date except on the 90 days before index date (initiators) and those whose current AED exposure started before the past 90 days (current users). This distinction ensures a relevant exposure time window as it is unlikely that an AED initiated shortly before the event would be responsible for the event based on the biological hypothesis. Moreover, it avoids a potential protopathic bias, especially when studying stroke, where starting an AED could be linked to early manifestations of cerebrovascular disease. Past users were patients whose last AED prescription ended between 365 and 90 days prior to the index date. Non-users were patients with no AED prescription in the year preceding the index date. Patients currently exposed to more than one AED class, for instance with prescriptions for both inhibiting and inducing AEDs, were classified into a separate category (multiple users). Consequently, current users, initiators, past users, multiple and non-users represented mutually exclusive exposure categories. In a subsequent analysis, patients currently exposed (based on the exposure definition above) were categorised according to duration of use during the entire follow-up from cohort entry to index date. The duration of continuous use of an AED was defined by successive prescriptions for the AED class of interest with a grace period of 90 days added to the end of a prescription to allow for refill time. If within the calculated duration a new prescription of the same AED class was issued, exposure was considered continuous, lasting until the last day of the last prescription. Duration of use was stratified into three groups according to the tertiles of the distribution of use in the controls.

\section{Covariates}

In addition to the inherent adjustment by the matching variables, the following potential confounders were included in the multivariable analysis: obesity (body mass index $\geq 30$ ), smoking status, alcohol abuse, transient ischaemic attack, diabetes, hypertension, hyperlipidaemia, atrial fibrillation, coronary artery disease, congestive heart failure, peripheral vascular disease, chronic obstructive pulmonary disease, chronic kidney disease, cancer, blood disorders and number of medical consultations. In addition, history of stroke was included in the analysis when studying MI and history of MI when studying stroke. All variables were measured 366-730 days prior to the index date and identified with relevant diagnostic codes except hypertension, diabetes and hyperlipidaemia which were defined using diagnostic codes or related medications. Medications prescribed within 1 year before the index date, such as oral anticoagulants, aspirin and other antiplatelet drugs, non-steroidal antiinflammatory agents, antidepressants, oral contraceptives and hormone replacement therapy, were also included as covariates. Missing data were expected for body mass index and smoking, so a separate category was created to classify this missing information; in a sensitivity analysis, we used multiple imputations for these missing values. We also performed a supplementary analysis excluding hyperlipidaemia as it is likely in the causal pathway between AED exposure and outcome. We also removed transient ischaemic attack or coronary artery disease from the potential confounders adjusted for in the multivariate model, depending on the outcome under study, as they may represent minor forms of the outcome of interest.

\section{Data analysis}

We calculated crude incidence rates for ischaemic stroke and MI, along with 95\% CIs based on the Poisson distribution. Incidence rate ratios (RRs) of ischaemic stroke and MI associated with current use of enzyme-inducing AEDs and enzyme-inhibiting AEDs relative to non-enzyme-inducing AEDs and their corresponding 95\% CIs were estimated from the ORs computed using conditional logistic regression. This approach provides unbiased estimates of the RRs that would be obtained from a Cox regression analysis, with little or no loss in precision, and is computationally more efficient, particularly when analysing time-varying exposures in large cohorts. ${ }^{24}$ In secondary analyses, we stratified cases and their matched controls according to the indication for AED to assess effect modification by the underlying reason for AED prescription. We also determined separately the risk of stroke and MI 
associated with current exposure to the different inducing AEDs as compared with non-inducing AEDs. Finally, we quantified the risk of stroke and MI according to the duration of AED use in an analysis restricted to current users ever exposed to one AED class only to exclude potential persistent effects of other AED classes.

We also calculated an approximation of absolute adjusted rate differences (RDs) and corresponding 95\% CIs as measures of the excess risk associated with the inducing AED. Adjusted absolute RDs were calculated as $\mathrm{R}_{0} \times(\mathrm{RR}-1)$, where $\mathrm{R}_{0}$ was the incidence rate of outcome among the non-inducing AED person time in the cohort (which was estimated to be $4.68 / 1000$ persons per year for ischaemic stroke and 3.61/1000 persons per year for MI), and RR was the adjusted RR for the inducing AED versus non-inducing AED.

Sensitivity analyses were performed to assess the robustness of our results. To explore the effect of potential misclassification of current exposure on the estimated RRs, the duration of the current exposure time window was shortened to 30 days prior to the index date for all cases and controls. Thus, some participants classified as currently exposed to AEDs at the index date based on the previous definition of current use in our main analysis became past or non-users. To assess the potential adjustment for covariates in the causal pathway, we repeated the primary analysis while adjusting for covariates measured at cohort entry. Finally, to assess the potential for residual confounding, we repeated the primary analyses (enzyme-inducing vs non-enzyme-inducing AED and enzyme-inhibiting vs non-enzyme-inducing AED) using propensity scores ${ }^{26}$ (PS) and high-dimensional propensity scores ${ }^{27}$ (hdPS). The PS were estimated in controls and applied to cases using covariates measured between 1 and 2 years before the index date. To ensure overlap in the distribution of PS for each of the exposure categories, $5 \%$ of cases and controls were excluded at either end of the distribution of the PS. HdPS were estimated in controls using an algorithm described in detail elsewhere. ${ }^{27}$ Briefly, after prioritising variables according to their potential to bias the exposure-outcome association, the 300 variables most likely to cause bias were included in the hdPS model. Estimated PS (or hdPS) were then included as quintiles in the conditional logistic regression model to assess the risk of stroke and MI associated with the different AED classes. CIs were calculated using a significance level of $5 \%$. All computations were performed using the SAS software V.9.3 (SAS Institute Inc, Cary, North Carolina, USA).

\section{RESULTS}

Of the 440191 patients with at least one AED prescription, 237050 were eligible for the stroke cohort and 241405 for the MI cohort (figure 1). The mean age at cohort entry in the stroke cohort was $56.1 \quad(\mathrm{SD}=17.5)$ years; $39.9 \%$ were males; and indications for AED prescription were epilepsy $(13.5 \%)$, pain $(49.8 \%)$, psychiatric disorders $(5.3 \%)$ and others/unknown (31.4\%). Characteristics were virtually the same in the cohort for studying MI. The mean duration of follow-up was 4.1 years $(\mathrm{SD}=3.9)$ in both cohorts, during which 4535 patients were diagnosed with ischaemic stroke and 3636 with MI, yielding overall incidence rates of $4.62(95 \%$ CI 4.49 to 4.75 ) and 3.63 (95\% CI 3.51 to 3.75 ) per 1000 persons per year, respectively. The characteristics of the cases of ischaemic stroke and MI and their matched controls are presented in table 1 . As expected, cases had a higher prevalence of vascular risk factors and comorbidities than controls.

In the stroke cohort, a total of $2743(60.5 \%)$ cases and $24971(57.4 \%)$ controls had received at least one AED prescription in the year before the index date. Among controls, $32 \%$ of these prescriptions were inducing AEDs (carbamazepine in 65\%), $18 \%$ were inhibiting AEDs and $49 \%$ were non-inducing AEDs (gabapentin in $58 \%$ ). The pattern of prescriptions was similar for the MI cohort. Among current users at index date, in the stroke outcome analysis, $85 \%$ of the cases and $87 \%$ of the controls remained exposed to the same class of AED during the entire follow-up, that is, they did not switch during follow-up. For MI, the numbers were $84 \%$ and $85 \%$ for cases and controls, respectively.

\section{Inducing AEDs}

Current use of inducing AED was associated with a small increased risk of ischaemic stroke (RR 1.16 (95\% CI 1.02 to 1.33)) relative to current use of non-inducing AED and no increased risk of MI (RR 1.12 (95\% CI 0.97 to 1.30 ); table 2). When stratified by indication, the increased risk of stroke was mainly driven by a $47 \%$ increased risk in the other/missing category (figure 2). The rate of stroke and MI did not vary according to the different inducing AED molecules (data not shown). When stratified by duration of use, there was no clear increased risk of ischaemic stroke with longer duration of current use of inducing AED (figure 2). However, there was an increased risk of MI with longer duration of use. Current inducing AED use of more than 24 months was associated with a $46 \%$ increased risk of MI (RR 1.46 (95\% CI 1.15 to 1.85)) compared with the same duration of non-inducing AED use, corresponding to an absolute adjusted RD of 1.39/1000 (95\% CI 0.33 to 2.45) persons per year.

\section{Inhibiting AED}

Current use of inhibiting AED was not associated with an increased risk of ischaemic stroke (RR 1.13 (95\% CI 0.96 to 1.33$)$ ) relative to current use of non-inducing AED and a non-statistically significant decreased risk of MI (RR 0.81 (95\% CI 0.66 to 1.00); table 2). When stratified by duration of use, there was no clear pattern of association between the risk of ischaemic stroke or 


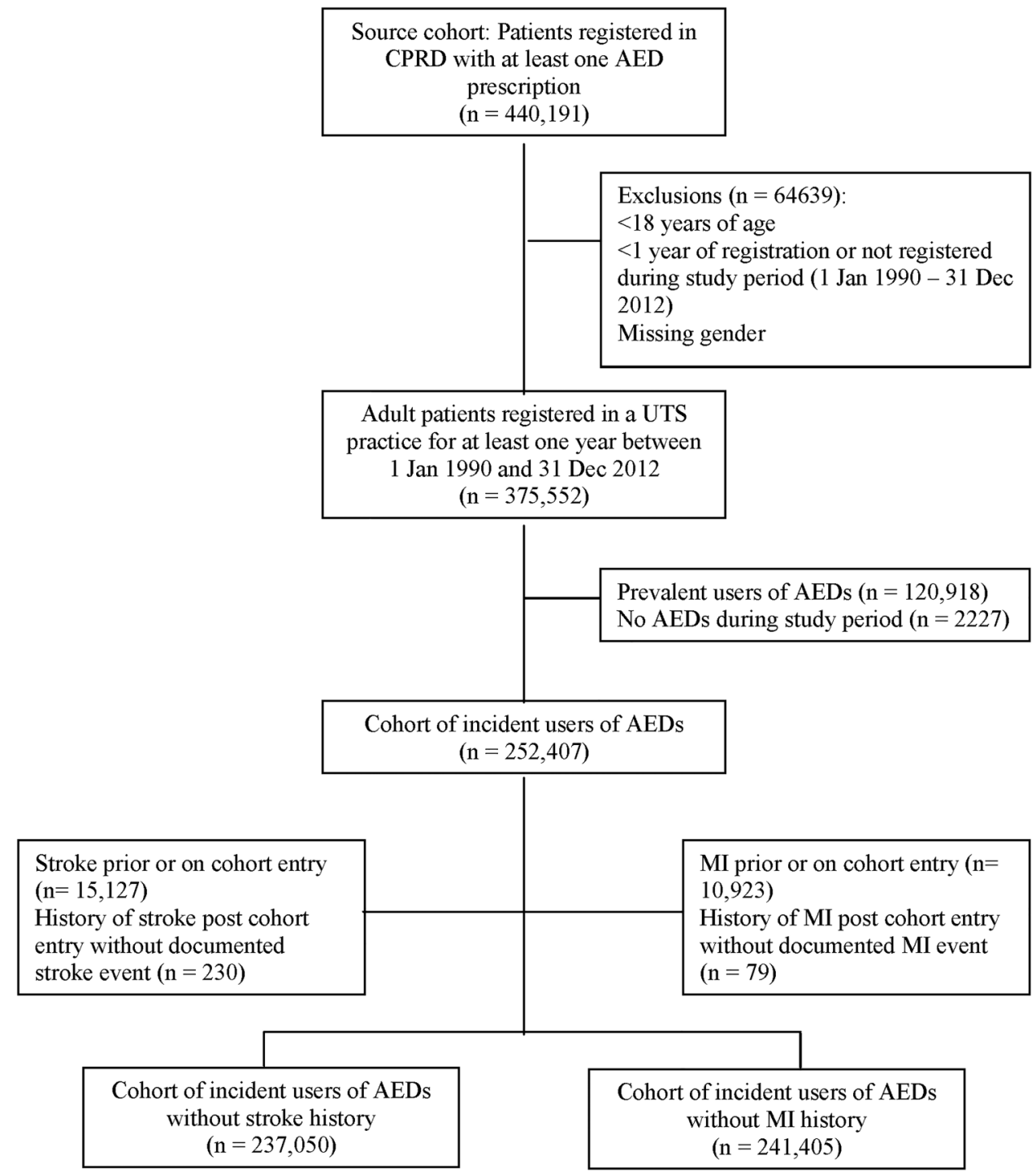

Figure 1 Details of cohorts definition. AED, antiepileptic drug; CPRD, Clinical Practice Research Datalink; MI, myocardial infarction; UTS, up-to-standard.

MI with longer duration of use of inhibiting AED (figure 3).

\section{Sensitivity analyses}

Restricting the exposure time window to 30 days did not affect the adjusted RRs of ischaemic stroke and MI estimated in the primary analysis (data not shown). Similarly, measuring covariates at cohort entry rather than 1-2 years before the index date, or controlling for confounding with PS or hdPS, did not materially change our results (figure 4). Restricting the analysis to ischaemic stroke only, that is, excluding strokes not otherwise specified, or using multiple imputations for missing data on smoking and body mass index, yielded similar estimates. Finally, adding dementia to the list of adjustment covariates and removing adjustment for transient ischaemic attack or coronary heart disease during follow-up resulted in very similar estimates.

\section{DISCUSSION}

Using a large population-based cohort of new users of AEDs, we showed no clear increased risk of ischaemic stroke with current use of inducing or inhibiting AEDs compared with non-inducing AEDs, except for a slight increased risk of ischaemic stroke associated with inducing AED, most likely due to residual confounding by indication. We found a small increased risk of MI with longer duration of inducing AED use. However, this relative increase would translate into few additional events and therefore a small clinical impact. Conversely, use of inhibiting AEDs was associated with a decreased risk of MI.

In the light of our results, the metabolic changes produced by highly inducing AEDs through the activation of the cytochrome P450 system do not lead to a clinically detectable increased risk of ischaemic stroke. The small increased risk of MI with longer duration of use but not ischaemic stroke may be related to the heterogeneity of 
Table 1 Characteristics of cases of ischaemic stroke and myocardial infarction and their matched controls

\begin{tabular}{|c|c|c|c|c|c|c|c|c|}
\hline \multirow[b]{2}{*}{ Variable } & \multicolumn{4}{|c|}{ Ischaemic stroke } & \multicolumn{4}{|c|}{ Myocardial infarction } \\
\hline & \multicolumn{2}{|c|}{$\begin{array}{l}\text { Cases } \\
\mathrm{N}=4533\end{array}$} & \multicolumn{2}{|c|}{$\begin{array}{l}\text { Controls } \\
\mathrm{N}=43514\end{array}$} & \multicolumn{2}{|c|}{$\begin{array}{l}\text { Cases } \\
\mathrm{N}=3636\end{array}$} & \multicolumn{2}{|l|}{$\begin{array}{l}\text { Controls } \\
\mathrm{N}=35372\end{array}$} \\
\hline Age at index date $($ mean $\pm S D)$ & $73.1 \pm 1$ & & $73.1 \pm 12$ & & $71.1 \pm 1$ & & $71.1 \pm 13$ & \\
\hline Follow-up time, in years $($ mean $\pm S D)$ & $3.7 \pm 3.6$ & & $3.7 \pm 3.6$ & & $3.8 \pm 3.4$ & & $3.8 \pm 3.4$ & \\
\hline Male-n (\%) & 1926 & $(42.5)$ & 18431 & $(42.5)$ & 1912 & $(52.6)$ & 18502 & $(52.6)$ \\
\hline \multicolumn{9}{|l|}{ Indication at cohort entry } \\
\hline Epilepsy-n (\%) & 870 & $(19.2)$ & 8115 & $(19.2)$ & 510 & $(14.0)$ & 4836 & (14.0) \\
\hline Pain-n (\%) & 2145 & (47.3) & 21029 & (47.3) & 1957 & $(53.8)$ & 19300 & $(53.8)$ \\
\hline Psychiatric disorders-n (\%) & 174 & (3.8) & 1341 & (3.8) & 106 & $(2.9)$ & 841 & (2.9) \\
\hline Other or missing-n (\%) & 1344 & (29.6) & 13029 & (29.6) & 1063 & (29.2) & 10395 & (29.2) \\
\hline \multicolumn{9}{|l|}{ Smoking status } \\
\hline Smoker-n (\%) & 2453 & $(54.1)$ & 21065 & $(47.7)$ & 2251 & $(61.9)$ & 17993 & $(50.5)$ \\
\hline Non-smoker-n (\%) & 1289 & $(28.4)$ & 13864 & $(32.0)$ & 901 & $(24.8)$ & 11118 & (31.4) \\
\hline Missing-n (\%) & 791 & $(17.4)$ & 8585 & (20.3) & 484 & (13.3) & 6261 & $(18.1)$ \\
\hline \multicolumn{9}{|l|}{ Obesity } \\
\hline Obese-n (\%) & 879 & $(19.4)$ & 8265 & $(18.6)$ & 823 & (22.6) & 7046 & (19.6) \\
\hline Non-obese-n (\%) & 2265 & $(50.0)$ & 21343 & $(48.7)$ & 1821 & $(50.1)$ & 17554 & (49.5) \\
\hline Missing-n (\%) & 1389 & $(30.6)$ & 13906 & (32.8) & 992 & (27.3) & 10772 & (30.9) \\
\hline \multicolumn{9}{|l|}{ Number of consultations } \\
\hline None-n (\%) & 916 & $(20.2)$ & 12955 & (29.8) & 777 & $(21.4)$ & 10391 & $(29.4)$ \\
\hline $1-3-n(\%)$ & 1139 & $(25.1)$ & 12061 & $(27.7)$ & 898 & $(24.7)$ & 9528 & (26.9) \\
\hline $4-6-n(\%)$ & 675 & (14.9) & 6252 & (14.5) & 547 & (15.0) & 5201 & (14.8) \\
\hline $7+$ n (\%) & 1803 & (39.8) & 12246 & $(28.1)$ & 1414 & (38.9) & 10252 & (28.9) \\
\hline Alcohol abuse-n (\%) & 277 & $(6.1)$ & 1836 & $(4.1)$ & 220 & $(6.1)$ & 1786 & $(5.0)$ \\
\hline Atrial fibrillation-n (\%) & 505 & (11.1) & 3131 & $(7.2)$ & 277 & (7.6) & 2465 & (7.1) \\
\hline Blood disorders-n (\%) & 23 & $(0.5)$ & 159 & $(0.4)$ & 17 & $(0.5)$ & 149 & $(0.4)$ \\
\hline Cancer/palliative care-n (\%) & 803 & (17.7) & 7274 & (16.7) & 635 & (17.5) & 5838 & $(16.6$ \\
\hline Heart failure $-n(\%)$ & 434 & $(9.6)$ & 2768 & $(6.7)$ & 323 & $(8.9)$ & 1723 & (5.1) \\
\hline Chronic kidney disease - n (\%) & 594 & (13.1) & 4941 & (11.4) & 550 & $(15.1)$ & 3921 & (11.2) \\
\hline COPD—n (\%) & 415 & $(9.2)$ & 3358 & $(7.7)$ & 454 & $(12.5)$ & 2668 & (7.5) \\
\hline Diabetes-n (\%) & 1028 & (22.7) & 6938 & $(15.7)$ & 975 & $(26.8)$ & 5855 & (16.4) \\
\hline Stroke-n (\%) & NA & NA & NA & NA & 442 & $(12.2)$ & 3046 & $(8.8)$ \\
\hline Myocardial infarction-n (\%) & 413 & $(9.1)$ & 3086 & $(7.2)$ & NA & NA & NA & NA \\
\hline Transient ischaemic attack一n (\%) & 589 & (13) & 3027 & $(7.2)$ & 427 & $(11.7)$ & 2770 & $(8.1)$ \\
\hline Hypertension-n (\%) & 2875 & $(63.4)$ & 24023 & $(55.1)$ & 2351 & $(64.7)$ & 19060 & (53.9) \\
\hline Ischaemic heart disease-n (\%) & 1201 & (26.5) & 9185 & $(21.2)$ & 996 & $(27.4)$ & 5569 & (15.8) \\
\hline Hyperlipidaemia-n (\%) & 1563 & (34.5) & 13744 & (30.9) & 1458 & $(40.1)$ & 11385 & (31.8) \\
\hline Pain-n (\%) & 2436 & $(53.7)$ & 22745 & $(51.8)$ & 2112 & $(58.1)$ & 19574 & $(55.1)$ \\
\hline Peripheral vascular disease $-\mathrm{n}(\%)$ & 509 & (11.2) & 2881 & $(6.7)$ & 499 & (13.7) & 2352 & $(6.7)$ \\
\hline Antihypertensive medications & 2600 & $(57.4)$ & 22078 & $(50.5)$ & 2166 & $(59.6)$ & 17479 & (49.3) \\
\hline ACE-n (\%) & 1106 & $(24.4)$ & 9022 & $(20.5)$ & 974 & $(26.8)$ & 7211 & (20.3) \\
\hline ARBs/RI-n (\%) & 420 & $(9.3)$ & 3739 & $(8.4)$ & 385 & $(10.6)$ & 3036 & $(8.5)$ \\
\hline$\beta$-Blockers-n (\%) & 1037 & (22.9) & 8614 & $(19.6)$ & 796 & (21.9) & 6476 & (18.2) \\
\hline Calcium channel blockers-n (\%) & 1152 & $(25.4)$ & 9026 & (20.6) & 1065 & (29.3) & 7369 & (20.7) \\
\hline Thiazide diuretics-n (\%) & 926 & (20.4) & 7968 & (18.2) & 715 & (19.7) & 6495 & (18.3) \\
\hline Diuretics-n (\%) & 124 & $(2.7)$ & 833 & $(1.9)$ & 99 & (2.7) & 610 & $(1.8)$ \\
\hline Loop diuretics-n (\%) & 1028 & (22.7) & 7457 & $(17.6)$ & 847 & (23.3) & 5196 & (15.0) \\
\hline Antidepressants- $\mathrm{n}(\%)$ & 1811 & $(40.0)$ & 15248 & (34.8) & 1407 & (38.7) & 12541 & (35.3) \\
\hline Antipsychotics-n (\%) & 794 & $(17.5)$ & 5190 & $(12.4)$ & 465 & $(12.8)$ & 3773 & $(11.0)$ \\
\hline HRT†-n (\%) & 195 & (7.5) & 2347 & $(9.2)$ & 153 & (8.9) & 1708 & (10.1) \\
\hline Oral anticoagulants- $\mathrm{n}(\%)$ & 450 & (9.9) & 2964 & $(6.7)$ & 294 & $(8.1)$ & 2511 & $(7.0)$ \\
\hline Antiplatelet agents- $\mathrm{n}(\%)$ & 2317 & (51.1) & 14813 & (34.1) & 1689 & $(46.5)$ & 11513 & (32.8) \\
\hline NSAIDs-n (\%) & 1222 & $(27.0)$ & 11786 & $(26.8)$ & 1107 & (30.4) & 9675 & (27.2) \\
\hline
\end{tabular}




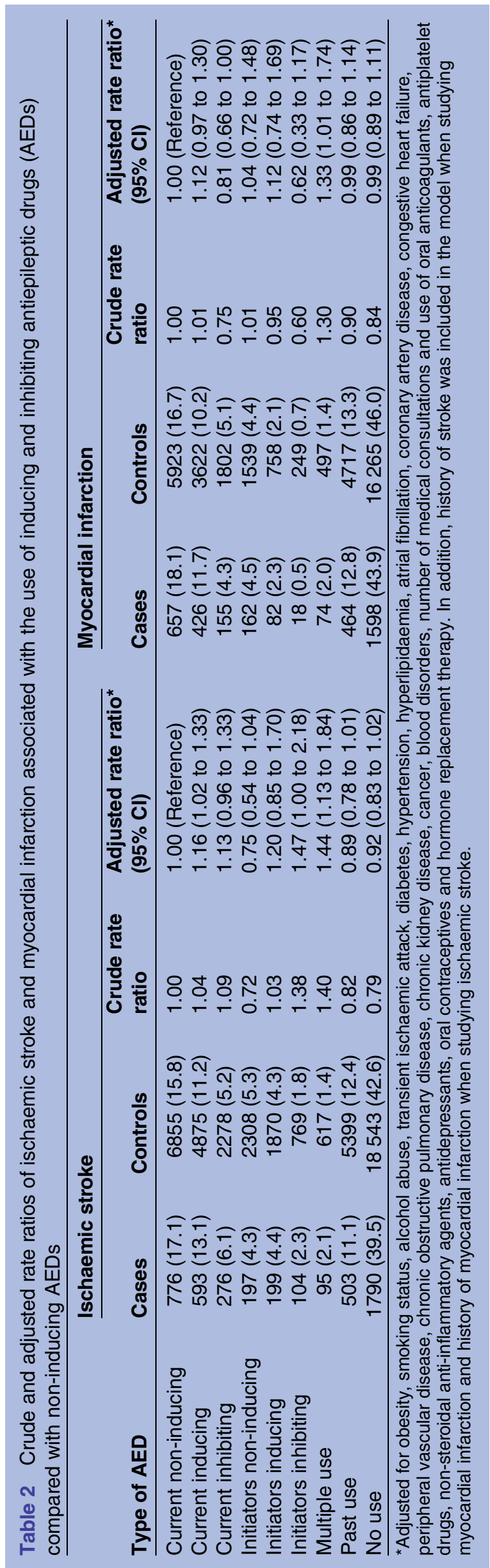

ischaemic stroke including various aetiological subtypes. Conversely, MI is almost always caused by atherothrombosis and the association between high serum lipid levels and cardiovascular risk has been clearly established. $^{28} 29$ The vascular effect of sodium valproate, which inhibits the cytochrome $\mathrm{P} 450$ pathway, is unclear. Indeed, valproate could have a proatherogenic effect through induction of insulin resistance, body weight gain, metabolic syndrome and increased oxidative stress. ${ }^{11} 12 \quad 30-33$ On the other hand, several studies suggest that valproate use is associated with lower LDL and total cholesterol serum levels. ${ }^{34}$ Moreover, studies in rodents have shown that sodium valproate attenuates the development of atherogenesis in hyperglycaemic mice via inhibition of glycogen synthase kinase-3, a serine/ threonine kinase which plays a central role in many cellular processes. ${ }^{35} 36$ Inhibition of glycogen synthase kinase-3 is also a promising target for cardioprotection. ${ }^{37}$ The capacity of valproate to affect other intracellular enzymes and pathways such as histone deacetylase, which plays a critical role in cardiac pathology, could also lead to cardioprotective effects. Indeed, administration of valproate had a beneficial effect on left ventricular remodelling after MI in rats via inhibition of histone deacetylase activity. ${ }^{38}$ Valproate may also exert a protective effect on large vessel ischaemic stroke via inhibition of histone deacetylase. Indeed, a genetic association between a variant of HDAC9 (encoding histone deacetylase 9) and this stroke subtype was recently identified. ${ }^{39}$ However, we did not have information on ischaemic stroke subtype and this association may have been masked in our analyses.

Only one previous study addressed the risk of ischaemic arterial events according to AED enzymatic properties using a US Medicare database and found no clear increased risk. However, AEDs were classified into two categories only, with the enzyme-inhibiting AED (valproate) being classified with the non-inducing AEDs, and the mean duration of follow-up was only 4 months. ${ }^{40} \mathrm{In}$ a Danish cohort study of patients with epilepsy, those treated with AEDs were at increased risk of vascular events as compared with non-treated patients except for MI. ${ }^{41}$ In a secondary analysis, the authors examined the risk associated with few individual AEDs. Compared with carbamazepine, sodium valproate was associated with a decreased risk of stroke and MI, whereas oxcarbazepine and phenobarbital were associated with an increased risk of death, and oxcarbazepine was associated with an increased risk of stroke. Several methodological differences with our work are noticeable such as the inclusion of incident and prevalent AED users at cohort entry, the assessment of few individual AEDs rather than AEDs according to enzyme properties, and the lack of adjustment for some potential confounding factors such as smoking or obesity. Although no consistent trends were found with AED individually, their finding of an attenuated risk of MI with valproate use is compatible with our results. However, we only found a trend towards a 
Figure 2 Adjusted rate ratios of ischaemic stroke and myocardial infarction associated with current use of inducing AEDs compared with non-inducing AEDs, stratified by indication and duration of use. AED, antiepileptic drug; IE, inducing AED; INHE, inhibiting AED; NIE, non-inducing AED; $\mathrm{RR}$, rate ratio.

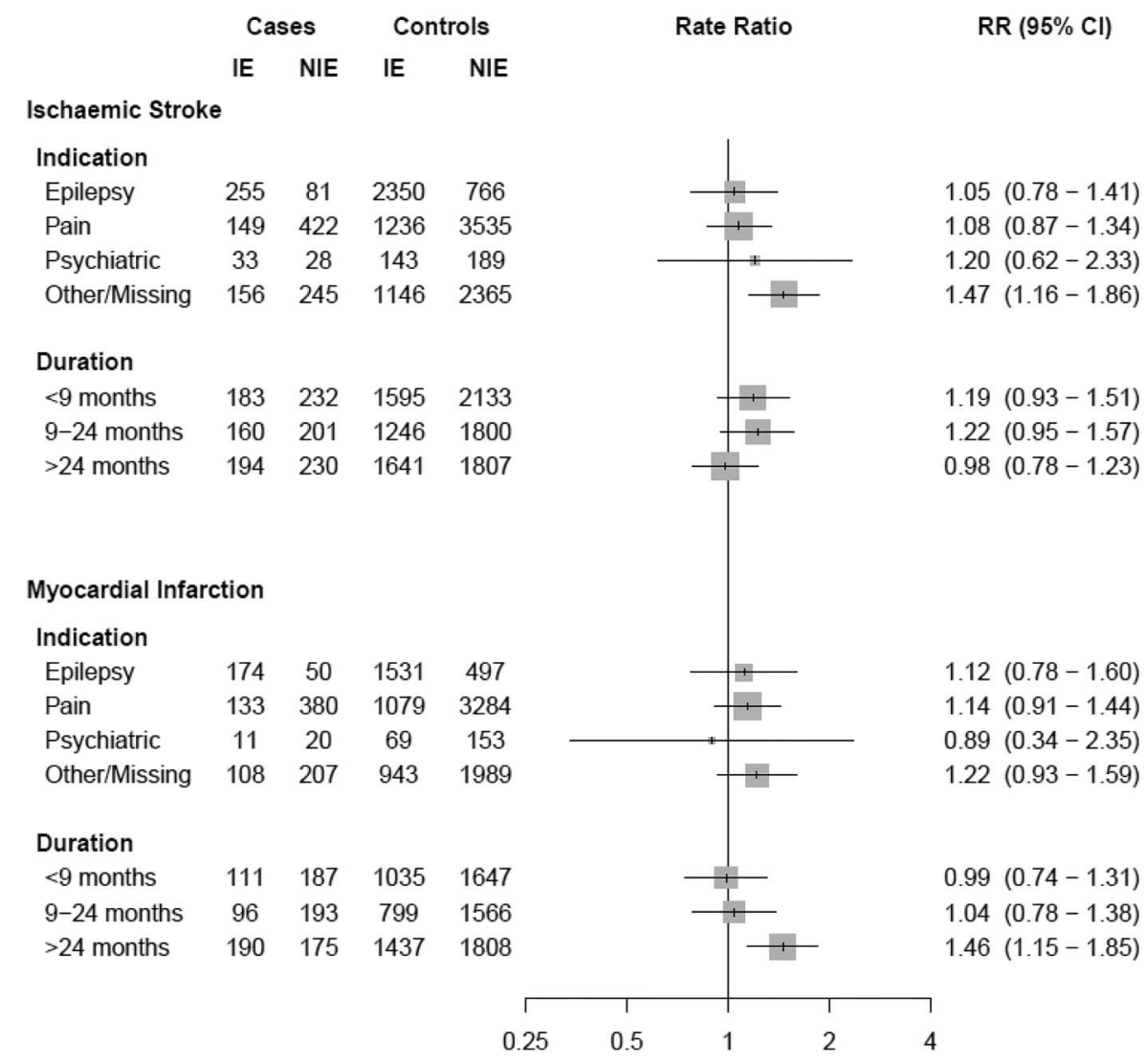

decreased risk for MI, which may be due in particular to the tighter adjustment for confounding in our study. One recent study using the CRPD database examined the risk of ischaemic stroke and MI associated with sodium valproate in a cohort of patients with epilepsy who were incident and prevalent users of one of the
Figure 3 Adjusted rate ratios of ischaemic stroke and myocardial infarction associated with current use of inhibiting AEDs compared with non-inducing AEDs, stratified by indication and duration of use. IE, inducing AED; INHE, inhibiting AED; NIE, non-inducing AED; $R R$, rate ratio.

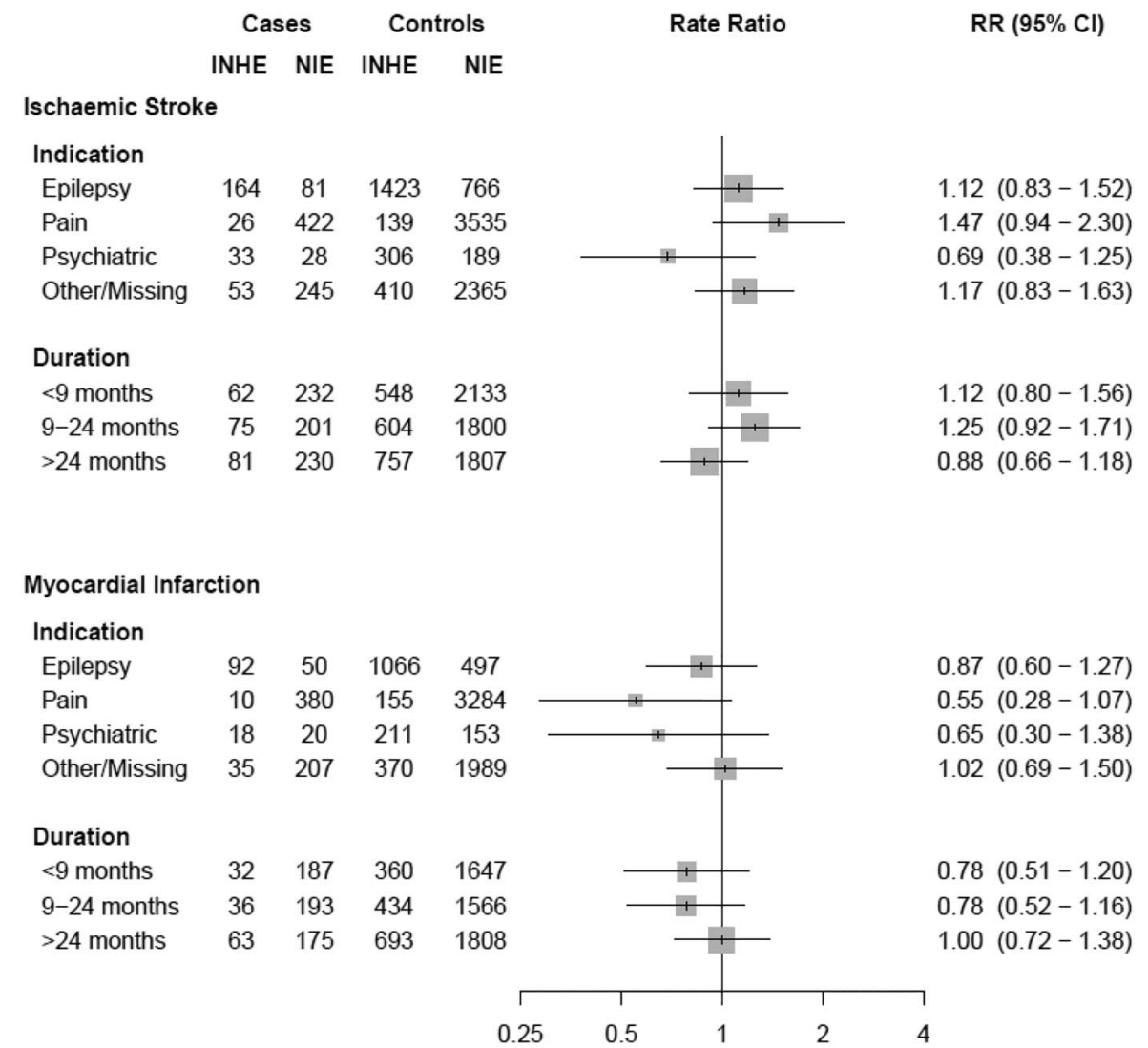


Figure 4 Adjusted rate ratios of ischaemic stroke and myocardial infarction associated with current use of inducing and inhibiting AEDs compared with non-inducing AEDs using different methods of adjustment. AED, antiepileptic drug; HDPS, high-dimensional propensity scores; IE, inducing AED; INHE, inhibiting AED; NIE, non-inducing AED; PS, propensity scores; RR, rate ratio.

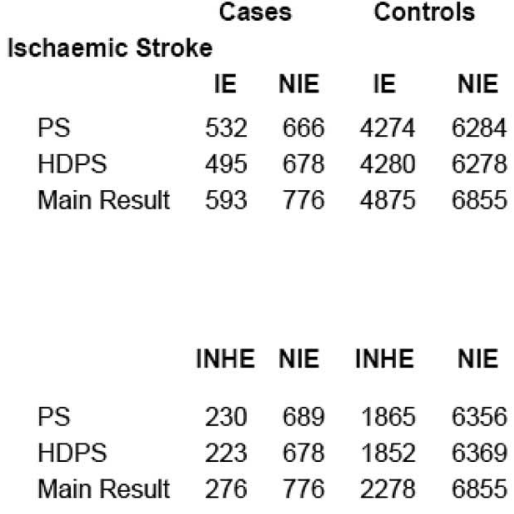

\begin{tabular}{lcccc}
\multicolumn{2}{l}{ Myocardial Infarction } & & & \\
& IE & NIE & IE & NIE \\
PS & 370 & 572 & 3157 & 5434 \\
HDPS & 362 & 558 & 3162 & 5429 \\
Main Result & 426 & 657 & 3622 & 5923
\end{tabular}

$\operatorname{RR}(95 \% \mathrm{Cl})$

$1.08(0.89-1.32)$

$1.14(0.93-1.39)$

$1.16(1.02-1.33)$

$1.19(0.92-1.53)$

$1.37(1.05-1.79)$

$1.13(0.96-1.33)$
$1.12(0.91-1.39)$

$1.22(0.98-1.52)$

$1.12(0.97-1.30)$ four following AEDs: sodium valproate, carbamazepine, phenobarbital and phenytoin. ${ }^{42}$ The authors reported a decreased risk of MI with sodium valproate compared with the use of other AEDs and a decreased risk of stroke with longer duration of use. However, the same trends were observed with the three other AEDs regarding the risk of stroke, and the results are most likely explained by several methodological issues as acknowledged by the authors. To the best of our knowledge, no previous study has assessed the risk of ischaemic events separately for the three AED categories while taking the indication for AED into account.

Our observational study was conducted in a populationbased setting, using the CPRD. The organisation of the UK healthcare system where over $99 \%$ of people are registered with a general practitioner ensures representativeness of the study population. The proposed design, consisting of a nested case-control approach within a well-defined cohort, virtually rules out the potential for selection bias. Also, the two outcomes, stroke and MI, have been extensively studied and well validated in the CPRD. Finally, since drug prescriptions are automatically recorded in the computerised file when issued by the physician, the definition of exposure is exempt of recall bias. However, it was based solely on prescriptions issued and not on prescriptions actually filled or taken by the patient, which could result in misclassification of exposure that is likely to be non-differential between cases and controls, therefore potentially biasing the results towards the null. The effect of potential misclassification was examined in sensitivity analyses which produced similar results. One of the potential threats to validity may be the presence of residual confounding due to differential drug prescription according to disease type or disease severity, that is, confounding by indication. By design, this bias was minimised by estimating the risk within groups of patients with the same indication and by choosing a treated comparator group. Indeed, treated patients are fundamentally different from non-treated patients in terms of disease severity, and using the latter group as a comparator would preclude disentangling the respective role of treatment and severity of the disease if the severity itself is associated with vascular events. Moreover, since the hypothesis is that the vascular risk varies with enzyme-inducing properties of AEDs, rather than with AEDs in general, a treated comparison group is more relevant. However, some residual confounding may still be present in the stratified analyses after matching by indication, but to various degrees within each indication. Still, our results were consistent within each matching category, except for the category with missing indication (for the stroke outcome) where we may expect a greater amount of residual confounding. This suggests that matching by indication was an efficient strategy to 
minimise confounding and that simply adjusting for numerous covariates in a multivariate model as carried out in some previous studies would not be sufficient. Of note, indication for a particular medication is not readily available in administrative databases either. Therefore, previous studies restricted their cohort to patients with epilepsy or simply adjusted for several comorbidities in the analyses. In theory, restriction is a good strategy, but given the incomplete information on indication in databases, this may lead to other biases such as selection bias. We were able to attribute AED prescription to a particular indication in up to $70 \%$ of our cohort, which permitted matching and possibly tighter adjustment. Moreover, we could assess that the risk was consistent in various indications, which was not possible in other studies. To further decrease the potential for residual confounding, all models were adjusted for several important potential confounders, including lifestyle variables such as smoking and alcohol abuse, which are usually not recorded in administrative databases and were not adjusted for in previous studies. Finally, we undertook several additional analyses using alternative methods to control for confounding such as PS and hdPS. We showed that, although the estimates varied slightly, particularly when adjusting using hdPS, the effect size remained consistent with a minor clinical impact.

In summary, we did not find a clear and consistent increased risk of ischaemic stroke with the use of inducing or inhibiting AEDs when compared with noninducing AEDs. We found an increased risk of MI with longer duration of use of inducing AEDs, which is most likely of small clinical importance. Conversely, use of inhibiting AEDs was associated with a decreased risk of MI, consistent with the underlying biological hypothesis.

\section{Author affiliations}

${ }^{1}$ Centre for Clinical Epidemiology, Lady Davis Institute for Medical Research, Jewish General Hospital, Montréal, Québec, Canada

${ }^{2}$ Department of Neurology and Neurosurgery, McGill University, Montréal, Québec, Canada

${ }^{3}$ Department of Epidemiology and Biostatistics, McGill University, Montréal, Québec, Canada

${ }^{4}$ Dalla Lana School of Public Health, University of Toronto, Toronto, Ontario, Canada

${ }^{5}$ Division of Clinical Epidemiology, Department of Medicine, McGill University, Montréal, Québec, Canada

Contributors CR designed the study. SD and J-FB critically reviewed the study protocol. SD analysed the data. OS participated in the statistical analysis. CR wrote the manuscript. All authors revised the manuscript. CR is the guarantor of the manuscript. All authors had full access to all of the data (including statistical reports and tables) in the study and can take responsibility for the integrity of the data and the accuracy of the data analysis.

Funding This study was funded by an operating grant (DRB-126643) from the Drug Safety and Effectiveness Network of the Canadian Institutes of Health Research (CIHR). CR is the recipient of a Chercheur-Boursier Award from the Fonds de la recherche en santé du Québec (FRSQ). KBF holds a New Investigator Award from the CIHR.

Competing interests $\mathrm{CR}, \mathrm{KBF}$ and J-FB report grants from the Canadian Institutes of Health Research (CIHR) during the conduct of the study.
Ethics approval The study protocol was approved by the Independent Scientific Advisory Committee of the CPRD (No. 14_181R) and the Research Ethics Committee of the Jewish General Hospital, Montreal, Canada; this protocol was made available to the journal reviewers.

Provenance and peer review Not commissioned; externally peer reviewed.

Data sharing statement No additional data are available.

Open Access This is an Open Access article distributed in accordance with the Creative Commons Attribution Non Commercial (CC BY-NC 4.0) license, which permits others to distribute, remix, adapt, build upon this work noncommercially, and license their derivative works on different terms, provided the original work is properly cited and the use is non-commercial. See: http:// creativecommons.org/licenses/by-nc/4.0/

\section{REFERENCES}

1. Rochat $P$, Hallas J, Gaist $D$, et al. Antiepileptic drug utilization: a Danish prescription database analysis. Acta Neurol Scand 2001;104:6-11.

2. Anderson GD. Pharmacogenetics and enzyme induction/inhibition properties of antiepileptic drugs. Neurology 2004;63(10 Suppl 4): S3-8.

3. Mintzer S. Metabolic consequences of antiepileptic drugs. Curr Opin Neurol 2010;23:164-9.

4. Bramswig S, Sudhop T, Luers C, et al. Lipoprotein(a) concentration increases during treatment with carbamazepine. Epilepsia 2003;44:457-60.

5. Calandre EP, Rodriquez-Lopez C, Blazquez A, et al. Serum lipids, lipoproteins and apolipoproteins $\mathrm{A}$ and $\mathrm{B}$ in epileptic patients treated with valproic acid, carbamazepine or phenobarbital. Acta Neurol Scand 1991:83:250-3.

6. Mintzer S, Skidmore CT, Abidin CJ, et al. Effects of antiepileptic drugs on lipids, homocysteine, and C-reactive protein. Ann Neurol 2009;65:448-56.

7. Nikolaos T, Stylianos G, Chryssoula N, et al. The effect of long-term antiepileptic treatment on serum cholesterol (TC, HDL, LDL) and triglyceride levels in adult epileptic patients on monotherapy. Med Sci Monit 2004;10:MT50-2.

8. Isojarvi JI, Pakarinen AJ, Myllyla VV. Serum lipid levels during carbamazepine medication. A prospective study. Arch Neurol 1993;50:590-3.

9. Mintzer S, Skidmore CT, Rankin SJ, et al. Conversion from enzyme-inducing antiepileptic drugs to topiramate: effects on lipids and C-reactive protein. Epilepsy Res 2012;98:88-93.

10. Mintzer S, Mattson RT. Should enzyme-inducing antiepileptic drugs be considered first-line agents? Epilepsia 2009;50(Suppl 8):42-50.

11. Isojarvi JI, Laatikainen TJ, Knip M, et al. Obesity and endocrine disorders in women taking valproate for epilepsy. Ann Neurol 1996;39:579-84.

12. Verrotti A, Scardapane A, Franzoni E, et al. Increased oxidative stress in epileptic children treated with valproic acid. Epilepsy Res 2008;78:171-7.

13. Gaitatzis A, Carroll K, Majeed A, et al. The epidemiology of the comorbidity of epilepsy in the general population. Epilepsia 2004;45:1613-22.

14. Garcia Rodriguez LA, Perez Gutthann S. Use of the UK General Practice Research Database for pharmacoepidemiology. Br J Clin Pharmacol 1998:45:419-25.

15. Walley T, Mantgani A. The UK General Practice Research Database. Lancet 1997;350:1097-9.

16. United Kingdom National Health Service. Read codes. Secondary United Kingdom National Health Service. Read codes. http://www. connectingforhealth.nhs.uk/systemsandservices/data/uktc/readcodes

17. Wood L, Martinez C. The general practice research database: role in pharmacovigilance. Drug Saf 2004;27:871-81.

18. Jick H, Jick SS, Derby LE. Validation of information recorded on general practitioner based computerised data resource in the United Kingdom. BMJ 1991;302:766-8.

19. Jick SS, Kaye JA, Vasilakis-Scaramozza C, et al. Validity of the general practice research database. Pharmacotherapy 2003:23:686-9.

20. Khan NF, Harrison SE, Rose PW. Validity of diagnostic coding within the General Practice Research Database: a systematic review. Br J Gen Pract 2010;60:e128-36.

21. Breslow NE, Day NE. Fitting models to continuous data. Statistical methods in cancer research, vol 2 the design and analysis of cohorts studies. Lyon, France: International Agency for research on Cancer, 1987:178-229. 
22. Suissa S. Novel approaches to pharmacoepidemiology study design and statistical analysis. In: Strome BL, ed. Pharmacoepidemiology. New York, NY: John Wiley \& Sons, Ltd, 2000:785-805.

23. Essebag V, Genest J Jr, Suissa S, et al. The nested case-control study in cardiology. Am Heart J 2003;146:581-90.

24. Essebag V, Platt RW, Abrahamowicz M, et al. Comparison of nested case-control and survival analysis methodologies for analysis of time-dependent exposure. BMC Med Res Methodol 2005;5:5.

25. Ray WA. Evaluating medication effects outside of clinical trials: new-user designs. Am J Epidemiol 2003;158:915-20.

26. D'Agostino RB Jr. Propensity score methods for bias reduction in the comparison of a treatment to a non-randomized control group. Stat Med 1998;17:2265-81.

27. Schneeweiss S, Rassen JA, Glynn RJ, et al. High-dimensional propensity score adjustment in studies of treatment effects using health care claims data. Epidemiology 2009;20:512-22.

28. Patel A, Woodward M, Campbell DJ, et al. Plasma lipids predict myocardial infarction, but not stroke, in patients with established cerebrovascular disease. Eur Heart J 2005;26:1910-15.

29. Yusuf S, Hawken S, Ounpuu S, et al. Effect of potentially modifiable risk factors associated with myocardial infarction in 52 countries (the INTERHEART study): case-control study. Lancet 2004;364:937-52.

30. Biton V, Mirza W, Montouris G, et al. Weight change associated with valproate and lamotrigine monotherapy in patients with epilepsy. Neurology 2001;56:172-7.

31. Kim JY, Lee HW. Metabolic and hormonal disturbances in women with epilepsy on antiepileptic drug monotherapy. Epilepsia 2007;48:1366-70.

32. Pylvanen V, Knip M, Pakarinen A, et al. Serum insulin and leptin levels in valproate-associated obesity. Epilepsia 2002;43:514-17.

33. Verrotti A, Manco R, Agostinelli S, et al. The metabolic syndrome in overweight epileptic patients treated with valproic acid. Epilepsia 2010;51:268-73.
34. Lopinto-Khoury C, Mintzer S. Antiepileptic drugs and markers of vascular risk. Curr Treat Options Neurol 2010;12:300-8.

35. Bowes AJ, Khan MI, Shi Y, et al. Valproate attenuates accelerated atherosclerosis in hyperglycemic apoE-deficient mice: evidence in support of a role for endoplasmic reticulum stress and glycogen synthase kinase-3 in lesion development and hepatic steatosis. Am J Pathol 2009;174:330-42.

36. Kim AJ, Shi Y, Austin RC, et al. Valproate protects cells from ER stress-induced lipid accumulation and apoptosis by inhibiting glycogen synthase kinase-3. J Cell Sci 2005;118(Pt 1):89-99.

37. Juhaszova M, Zorov DB, Yaniv $\mathrm{Y}$, et al. Role of glycogen synthase kinase-3beta in cardioprotection. Circ Res 2009;104:1240-52.

38. Lee TM, Lin MS, Chang NC. Inhibition of histone deacetylase on ventricular remodeling in infarcted rats. Am J Physiol Heart Circ Physiol 2007;293:H968-77.

39. Bellenguez C, Bevan S, Gschwendtner A, et al., International Stroke Genetics Consortium (ISGC), Wellcome Trust Case Control Consortium 2 (WTCCC2). Genome-wide association study identifies a variant in HDAC9 associated with large vessel ischemic stroke. Nat Genet 2012;44:328-33.

40. Patorno E, Glynn RJ, Hernandez-Diaz S, et al. Risk of ischemic cerebrovascular and coronary events in adult users of anticonvulsant medications in routine care settings. J Am Heart Assoc 2013;2 e000208.

41. Olesen JB, Abildstrom SZ, Erdal J, et al. Effects of epilepsy and selected antiepileptic drugs on risk of myocardial infarction, stroke, and death in patients with or without previous stroke: a nationwide cohort study. Pharmacoepidemiol Drug Saf 2011;20: 964-71.

42. Dregan A, Charlton J, Wolfe CD, et al. Is sodium valproate, an HDAC inhibitor, associated with reduced risk of stroke and myocardial infarction? A nested case-control study. Pharmacoepidemiol Drug Saf 2014;23:759-67. 